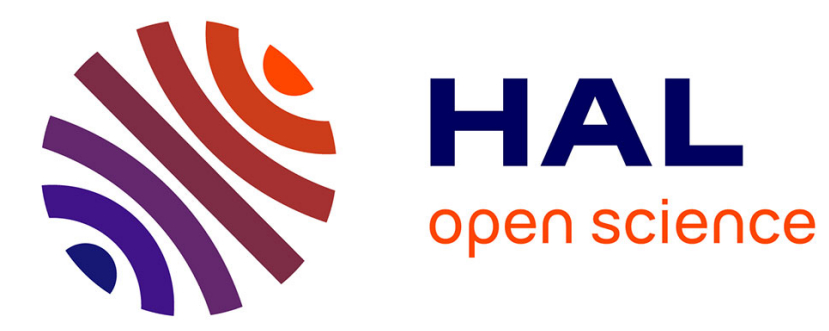

\title{
The copycat solution to the shadow correspondence problem
}

\author{
Roberto Casati
}

\section{To cite this version:}

Roberto Casati. The copycat solution to the shadow correspondence problem. Perception, 2007, pp.000-000. ijn_00171287

\section{HAL Id: ijn_00171287 \\ https://hal.science/ijn_00171287}

Submitted on 12 Sep 2007

HAL is a multi-disciplinary open access archive for the deposit and dissemination of scientific research documents, whether they are published or not. The documents may come from teaching and research institutions in France or abroad, or from public or private research centers.
L'archive ouverte pluridisciplinaire HAL, est destinée au dépôt et à la diffusion de documents scientifiques de niveau recherche, publiés ou non, émanant des établissements d'enseignement et de recherche français ou étrangers, des laboratoires publics ou privés. 
Roberto Casati

Institut Nicod

CNRS-EHESS-ENS

Paris, France

and

Università IUAV

Venice, Italy 


\section{The copycat solution to the shadow correspondence problem}

Cast shadows (henceforth simply 'shadows') depicted in artworks can fulfil some of their perceptual roles, such as helping the retrieval of $3 \mathrm{~d}$ structure and of relative positions of objects, without having to be geometrically or physically accurate. The visual system displays a wide tolerance for shadows that are mostly inaccurate, at times making use of the scarce accurate but relevant information that can still be retrieved from them. However, the extent of the tolerable inaccuracy is at present still unexplored. We propose that inaccuracy can be not only totally acceptable but in some cases is even likely to be preferable to accuracy if the main perceptual role of shadows is to help locate in a scene the objects that cast them.

We examined a small but effective historical corpus of pictorial endeavours, from which it appears that in some cases painters have used a copycat strategy for drawing the terminator of a shadow, i.e. they have produced a replica of the visible profile of the caster, which in the norm yields an impossible shadow. The copycat strategy is perceptually effective for solving the correspondence problem of associating a shadow with its caster; copycatting can be more effective than other solutions, including the depiction of the geometrically correct shadow, and is complementary to coarser solutions to the correspondence problem.

These phenomena provide insight into the computations effectively used by the brain to deal with space perception. In particular, the brain is not relying on some sort of simplified physical model of the world, for the shadows produced by the copycat effect would correspond to a more complicated physical situation than the one encountered in real life. 
Cast shadows (henceforth simply 'shadows') depicted in artworks can fulfil some of their perceptual roles, such as helping the retrieval of $3 \mathrm{~d}$ structure and of relative positions of objects, without having to be geometrically or physically accurate, and while at times being grossly distorted, inconsistent, or physically and geometrically impossible. (Casati 2000, Casati 2006, Mamassian 2004; Jacobson and Werner 2004; and Mamassian et al. 1996 for a similar observation about self shadows.) More generally, the visual system displays a wide tolerance for shadows that are mostly inaccurate, at times making use of the scarce accurate but relevant information that can still be retrieved from them. For instance, a shadow whose shape is not geometrically correct but which is cast in an area where it roughly belongs can be used to retrieve the relative positions of the caster and the surface of projection.

In being tolerant, the visual system acts parsimoniously, as shadows are ephemeral components of the visual scene, and the costs of computing their accuracy are too high in the face of benefits that can be reaped anyway through the use of less accurate representations.

However, the extent of the tolerable inaccuracy is at present still unexplored. We propose that inaccuracy can be not only totally acceptable but in some cases is even likely to be preferable to accuracy if the main perceptual role of shadows is to help locate in a scene the objects that cast them.

We examined a historical corpus of pictorial endeavours, from which it appears that in some cases painters have used what we term a copycat strategy for drawing the terminator of a shadow, i.e. they have produced a replica of the visible profile of the caster, which in the norm yields an impossible shadow. The examples show that the copycat strategy is perceptually effective for solving the 
correspondence problem of associating a shadow with its caster, and that copycatting can be more effective than other solutions, including the depiction of the geometrically correct shadow.

These phenomena provide insight into the computations effectively used by the brain to deal with space perception. In particular, the brain is not relying on some sort of simplified physical model of the world (Cavanagh 2005), for the shadows produced by the copycat effect would correspond to a more complicated physical situation than the one encountered in real life.

The perceptual retrieval of spatial organization via inferences concerning cast shadows is a well-documented phenomenon. Shadows are normally reliable position indicators (Mamassian et al. 1998; Kersten et al. 1996). They provide a precious cue as to the location of the shadow caster relative to the surface the shadow is cast upon, and thereby relative to neighbouring surfaces. In order to so function, a specific region in the image must be labelled as a shadow (call this the labelling problem) and the shadow region must be linked to the object that casts it (call this the correspondence problem). (Mamassian 2004) describes a coarse solution to the correspondence problem, based on matching the centre of mass of the caster with the centre of mass of the shadow, and ignoring details such as the actual shape of the shadow. The coarse solution explains tolerance for some impossible shadows: if actual details are not taken into account, then little does it matter if a pictorial representation of a shadow is accurate. However, the coarse solution to the correspondence problem only explain tolerance for shadows that are globally consistent with light direction and are inaccurate as to their matching of the caster's features. 
We describe a pictorial strategy for solving the correspondence problem that is complementary to the one discussed by Mamassian, as it yields as a solution shadows that are inconsistent with light direction but which are accurate as to their matching of the caster's features. The strategy has actually been discovered and used by painters in their attempts to depict shadows (it is taken here to count as a psychological discovery). Pictorial interest in shadows has spanned the Renaissance, which can be seen as a period of intense experimentation in pictorial solutions to space organization problems. Indeed, Reinassance painters used sophisticated perspective constructions and had a mastery of chiaroscuro (shading) effects, but for about a century they failed to realize that shadows are just chiaroscuro in perspective, and ended up producing a number of geometrically incorrect shadows in a trial-and-error fashion. The problem of shadow depiction arises as shadows are in general difficult to paint, for the reduced light contrast available on the canvas makes it easy to come up with a result that would make shadows appear not as lighting accidents but as permanent patches of dark colour. In Leonardo Da Vinci's own terminology, a poorly represented shadow would appear 'wooden' (quoted in Da Costa Kauffman 1975; see Gombrich 1995, Stoichita 1997, Baxandall 1995). Incidentally, it may appear that a solution to the labelling problem should precede any solution of the correspondence problem, i.e. that an observer should first discern something as a shadow before working out what it is a shadow of. However, if it were possible to automatically establish a correspondence between the outline of an object and the profile of a dark patch that could be the concerned shadow, then the labelling problem would be solved via a solution of the correspondence problem. The dark patch would be labelled a shadow precisely because as such it provides a solution to the correspondence problem. 
Shadows are images of their casters, and a simple way to solve the correspondence problem is to remind the viewer of this fact. This can be done just by making the caster recognizable in its shadow. There is ecological support for this phenomenon in particular lighting conditions. For instance, when the sun is close to the horizon, shadows e.g. of people cast on vertical walls can be particularly informative, as opposed to shadows cast on the ground by zenithal light, which collapse the figure of the caster. A very effective way to make the caster recognizable in the shadow is to simply imitate the visible profile of the caster with the terminator line of the shadow. Call this the copycat effect.

One of the earliest examples of the copycat phenomenon is to be found in an illumination of the 14th century representing the Biblical episode of the escape of the Spies of Jericho. In the Bible (Joshua 1,15) the gentile prostitute Rahab helps two men sent by Joshua as spies to Jericho to escape on a rope from the roof of her house. The episode was illustrated in the so called Visconti Hours (Florence, Biblioteca Nazionale, BR 397, LF 22) by Belbello da Pavia, a painter of the first half of the $15^{\text {th }}$ century (Meiss 1972).

The artist has depicted a shadow of the two spies on the wall. This addition is obviously the result of precise deliberation, as the shadow is very complex, imitates the two actors, and is detached from them (as distinct from the quickand-dirty almost amorphous shadows that painters attach at the feet of represented actors to signal the actors' position). An informal survey has shown that observers have no difficulty in interpreting the shadow as a shadow of the two spies, and in fact find it a rather compelling shadow (Fig. 1). 


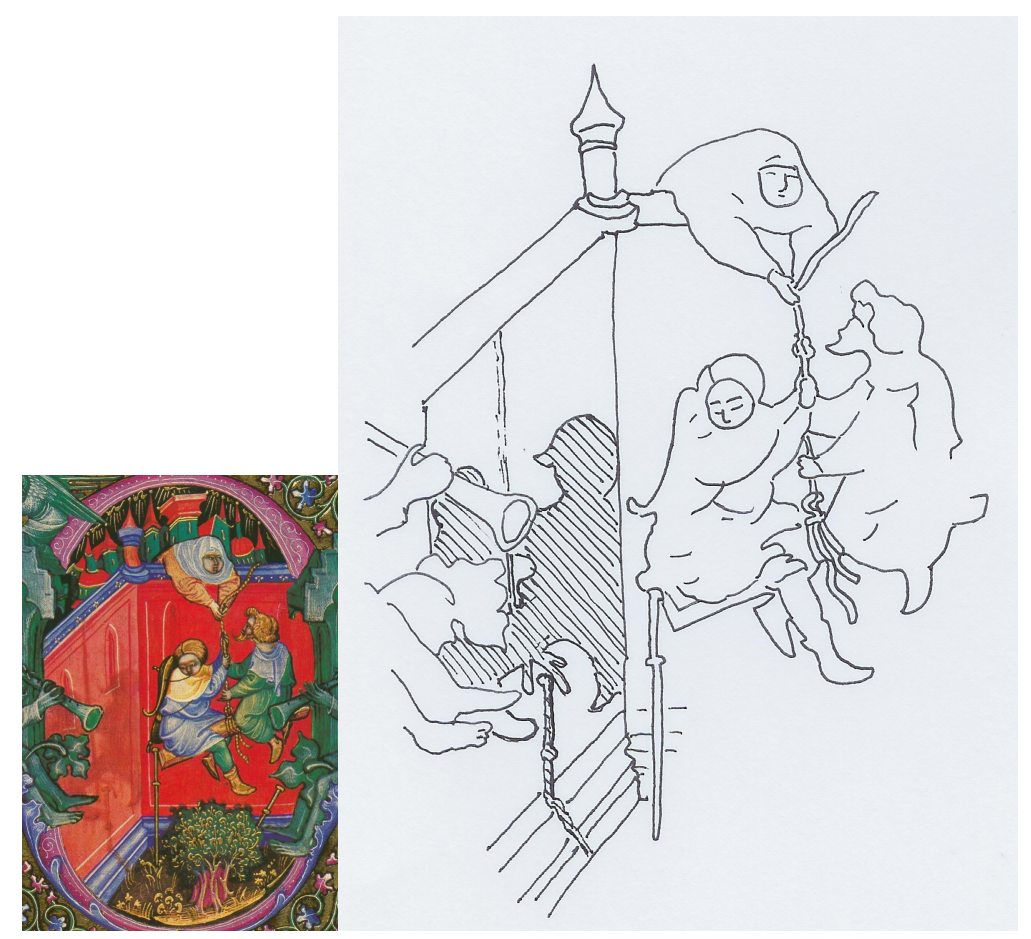

Fig. 1 Belbello da Pavia, The spies of Jericho escape, in the Visconti Hours (probably 1420-1425).

Note the copycat shadow on the left wall.

Now, the shadow under consideration has an interesting feature. Its profile copies the profile of the visible silhouette of the pair of spies (the copycat effect). That is, it has the profile of the shadow that the two actors would cast had the source of light been aligned with them and the observer. But of course, any source of 
light in such a position would normally produce a shadow that is totally hidden behind the actors, and normally no such source would produce a shadow that has such a profile and is visible on the side of the actors. Hence this particular shadow is incorrect: either it should not be visible, blocked from view by the casters, or it should have a very different profile. However, this mistake is hardly noticed; the effect is not that of the perception of an impossible figure, with an associated sense of wrongness. Moreover, knowing about the mistake does not make one see the shadow as impossible: the effect is resilient to knowledge. Finally, the shadow appears to be not only acceptable, but compelling, in particular when it comes to associating it to an object.

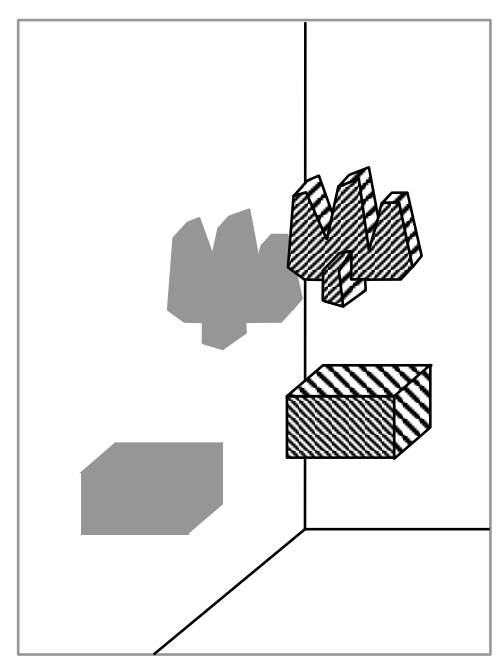

Fig. 2 Two schematic examples of the copycat effect. The

bottom object is seen as being closer to the viewer than the top object, as the top object is closer to its shadow on the left wall, and the shadow of the bottom object is closer to the observer than the shadow of the top object.

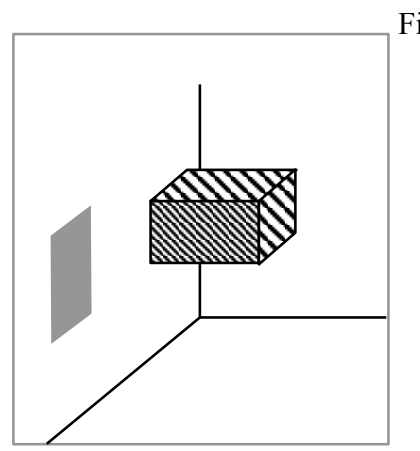

Fig. 3 A projectively correct shadow for the parallelepiped

displayed in fig. 2, left. Light source at infinity. 

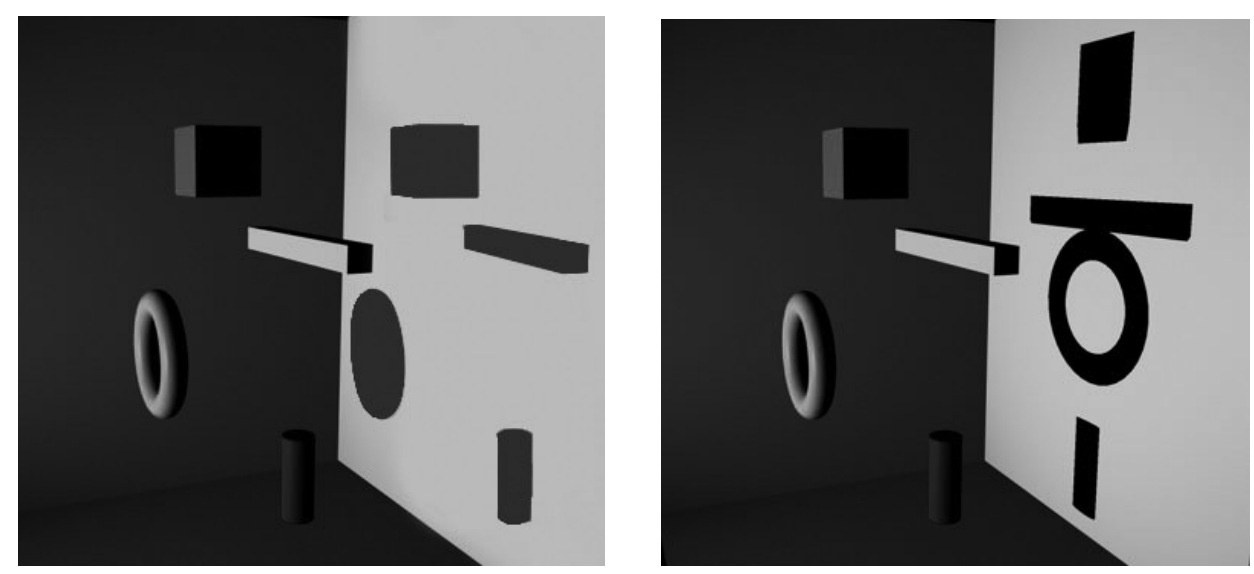

Fig 4. Copycat (left) and real shadows (right) of four solid objects. Digital images by Jelena Arsenic and Svetislava Isakov.

A study of a corpus of shadow depictions from the early Renaissance of roughly 1500 items indicates that in a few cases (around 15) the cast shadows clearly imitate the shadow of the caster (where imitation is to be understood here in the weak sense in which the observer can recognize that the shadow is a shadow of the caster by looking at the shadow's profile). Of these, about $1 / 3$ display the condition of the copycat effect. This period has been circumscribed because no mathematical theory and algorithms of shadow depiction were available to early Renaissance painters, who were nevertheless interested in depicting cast shadows and provided a number of cognitively intriguing attempts to do so. (Casati 2004)

The examples include shadows present in the following paintings: 
Giovanni di Paolo (ca. 1403-1482), S. Francis, Siena, Pinacoteca; Giovanni di Paolo, Flight Into Egypt, Siena, Pinacoteca; Paolo Uccello (1397-1475), Flood and Waters Subsiding (1447-48) Green Cloister, Santa Maria Novella, Florence (see fig. 5A); Carlo Crivelli (1430c-1492c), Annunciation (1486) London, National Gallery Carlo Crivelli, Mary and Child (1472-73), New York, Metropolitan Museum (see fig 5B).

For a later example, Giorgio Vasari (1511-1574) The origin of painting, Florence, Vasari House (1573) (see fig. 5C).

A
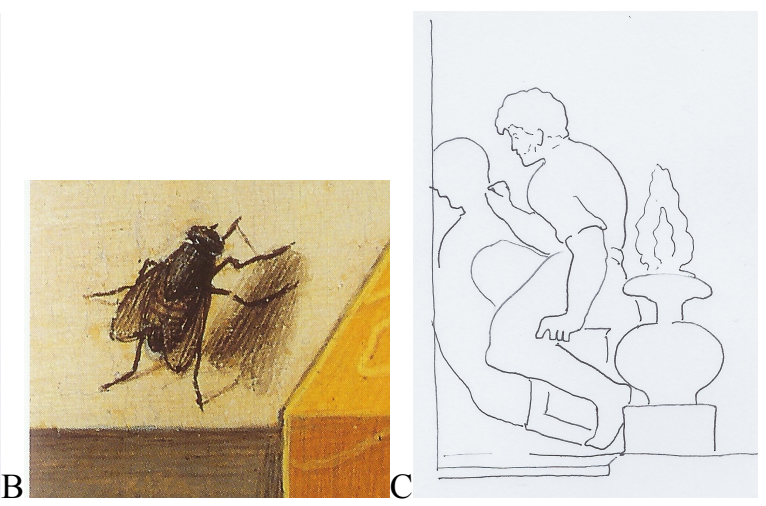

Fig 5

More faintly, the phenomenon is displayed in back panel, Hugo Van der Goes (1420?-1482), Portinari Triptich (1475), Florence, Uffizi; Roger van der Weyden (1400?-1464), Mary and St. Jean (after 1460), Philadelphia.

The work of Carlo Crivelli is rich of "innocent" copycat shadows which have not been counted in because they are cast either by two dimensional objects such as leaves or carpets (see the shadows at the top of the above quoted Mary with Child), or by cylindrical objects such as certain vegetables or keys (Beato Giacomo della Marca, Urbino, Galleria Nazionale).

Some of the pictures reproduced in Stoichita 1997, although from different artistic periods and techniques, are also worth mentioning: Samuel van Hoogstraten, The Shadow Dance, engraving, 1675, reproduced on p. 130; Jacques de Gheyn II, Three Witches Looking for Buried Treasure, pen and ink, 1604, on p. 134; Thomas Holloway et al., Machine for Drawing Solhouettes, engraving, 1792, on p. 156.

The copycat effect is unlikely to occur if the painter paints aiming at truth. In real-life situations copycat shadows can sometimes be seen on a frontal wall if cast by a light from almost behind the observer and by a relatively flat object lying in a frontoparallel plane; but this is not the general case. When the effect occurs, we can safely assume that the painter worked without consulting a model, that is, he generated the shadow out of his intuition of what the shadow should look like. In such cases the copycat mistake appears to be either a geometric 
reasoning mistake and/or a deliberate choice. Reasons for the deliberate choice could be found in the need to improve the plausibility of the shadow depiction, by making sure that the shadow is perceptually labelled as a shadow (solving the labelling problem), and making sure it is linked to the object that casts it (solving the correspondence problem).

The small epidemiology of the copycat effect is by no means limited to the early Renaissance. A dramatic example of the depiction of a cast shadow that is not geometrically correct yet is not only acceptable but preferable to the correct shadow shows up in a $20^{\text {th }}$ century masterpiece, Magritte's Empire of Light (of which several versions exist; see fig. 6).

(Magritte picture unavailable for copyright issues)

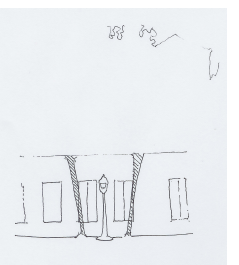

Fig. 6

René Magritte, Empire of Light (L'Empire des lumières), 1953-54. Oil on canvas, 195.4 x 131.2 cm. Peggy Guggenheim Collection. 76.2553 PG 102. René Magritte C 2003 C. Herscovici, Brussels/Artists Rights Society (ARS), New York.

The painting is usually taken as an illustration of a 'paradoxical' treatment of light: it is as if a daylight sky were juxtaposed to a dark night scene. The lower half of the painting is lit by a feeble streetlamp; the shadow of the streetlamp's casing is cast on the wall of the building (see the two dark oblique lines visible next to the central windows). Now, lamps such as the one depicted by Magritte are typically assembled from glass panes of equal size and shape. As these are 
isosceles trapezoids, the lamp is just a truncated pyramid. Hence the light is in the same plane, normal to the ground, as each of the edges of the pyramid. This entails that any intersection of this plane with a vertical wall (the shadow on the wall) is a vertical line (see fig. 7). Hence the shadow cast on the wall in Magritte's painting is incorrect: it should appear as a vertical line. And indeed, in real life situations (see fig. 8), similar streetlamps cast 'straight' shadows that are visually awkward. 


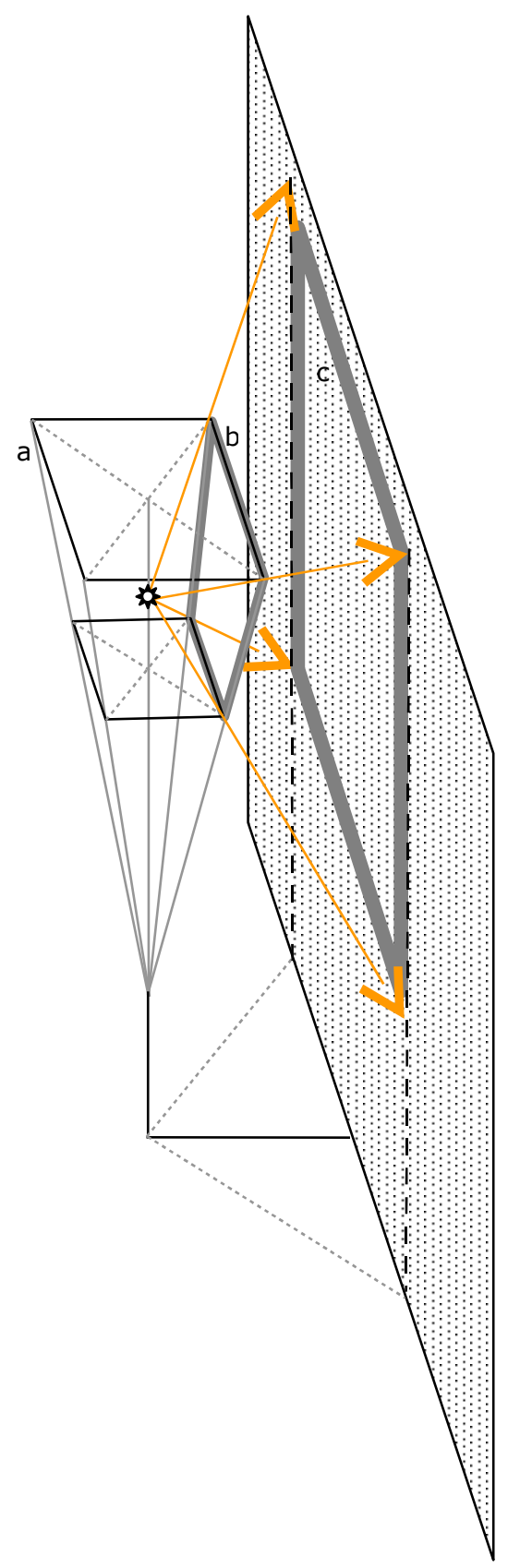


Fig. 7 How isosceles trapezoids, in certain conditions, can only project straight shadows. A schematic representation of a streetlamp (a), whose casing frame (b, thick outline) casts shadow (c) on a vertical wall.

However, in Magritte's painting the shadow, as it stands, is both recognized as a shadow and is assigned to the appropriate caster, i.e. the lamp.
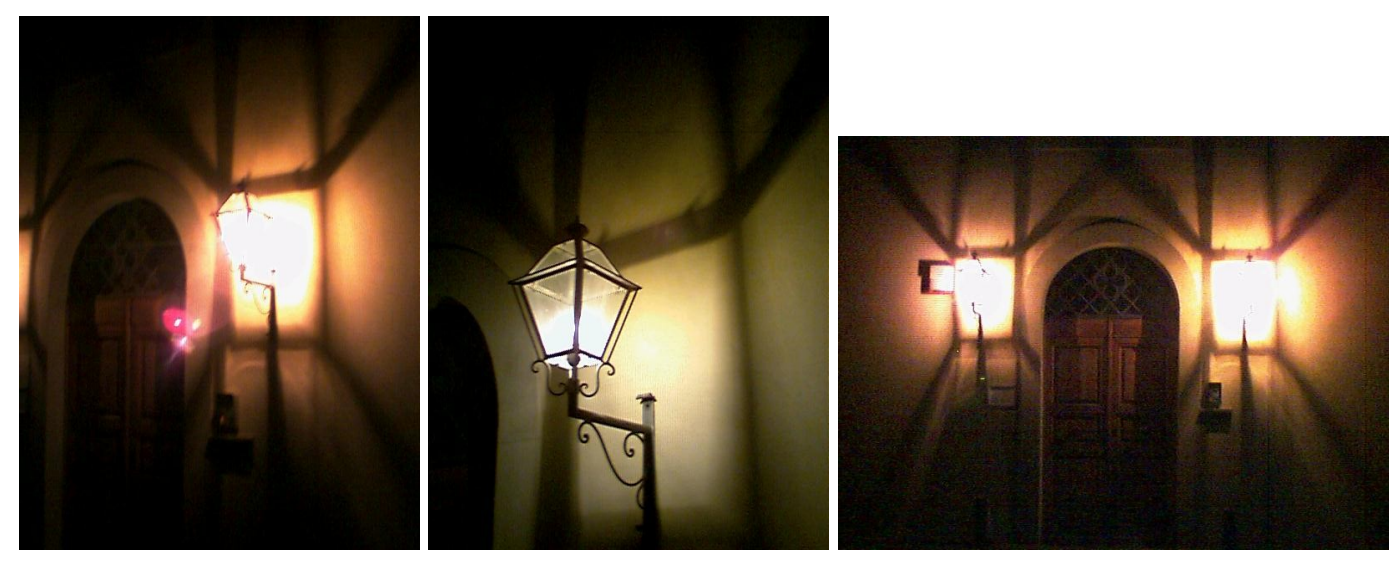

Fig. 8 Real life examples of streetlamps casting shadows of their case. Note that the shadow has something implausible about it, and appears to fail the correspondence test.|Pictures by the author.

In the Magritte example the copycat effect is realized through a similarity transform that preserves the visual shape through an enlargement. Once more, 
however, the choice of a mistaken shadow is shown to enhance the understanding of the picture by providing a quick solution to the correspondence problem.

\section{Conclusions}

The copycat effect and more in general various types of mistaken shadow depictions are used by artists to make a convincing impression on the viewer of a painting. The impression is convincing when two problems are solved: the shadow labelling problem of correctly categorizing a region in the visual field as a shadow, and the correspondence problem of associating the shadow to its caster. Solving the two problems reduces the noise in the picture, as doing so takes away attentional sources from the area corresponding to the shadow, an area that otherwise could be mistaken for an object owing to its strong luminance contrast with its surroundings.

The correspondence problems may not arise in such a dramatic form outside the pictorial, static $2 \mathrm{~d}$ setting. In dynamic situations, it would be easy to establish a correspondence between parts of a moving shadow and parts of the moving caster, as the movements would be synchronised and share a number of cinematic properties. Besides, the correspondence problem may arise only in very specific cases in ecological situations, where many shadows of many objects are visually available.

It has been surmised (Cavanagh 2005) that tolerance for certain types of mistaken shadows indicates that the visual system uses a sort of simplified physics in recognizing everyday scenes. However, the copycat effect suggests that something different should be the case. The physics underlying the copycat effect would have to be, if anything, much more complicated than the physical description of an ordinary environment, as light would have to bend in strange 
ways in order for most copycat shadows to be cast. Instead, the visual system is here using relatively low-level heuristics, such as shape-matching between an object's visible profile and a shadow, to economically solve the correspondence problem.

\section{References}

Baxandall M, 1995 Shadows and Enlightenment (New Haven: Yale UP)

Casati R, 2002 The Shadow Club (New York, Knopf)

Casati R, 2004 "Methodological Issues in the Study of the Depiction of Cast Shadows: A Case Study in the Relationships Between Art and Cognition" The Journal of Aesthetics and Art Criticism, 62, 2, 163-174

Casati R, 2006 “The cognitive science of holes and cast shadows” Trends in Cognitive Science, 10, 2, 54-55

Cavanagh P, 2005 “The Artist as Neuroscientist” Nature 434, 301-307

Da Costa Kaufmann T, 1975 “The Perspective of Shadows: The History of the Theory of Shadow Projection” The Journal of the Warburg and Courtauld Institutes, 38, 258-287

Gombrich E H, 1995 Shadows: the Depiction of Cast Shadows in Western Art (London: National Gallery/New Haven:Yale UP)

Jacobson J, Werner S, 2004 "Why cast shadows are expendable: Insensitivity of human observers and the inherent ambiguity of cast shadows in pictorial art" Perception, 33, 11, 1369 - 1383

Kersten D, Knill D C, Mamassian P, and Bülthoff I, 1996 “Illusory motion from shadows” Nature 379, 31

Mamassian P, 2004 "Impossible shadows and the shadow correspondence problem” Perception,

$2004,33,11,1279-1290$

Mamassian P, Kersten D, 1996 "Illumination, shading and the perception of local orientation” Vision Research, 35, 2351-

2367 
Mamassian P, Knill D C and Kersten D, 1998 "Perceiving spatial layout from cast shadows" Trends in Cognitive Science, 2

(8), 288-295

Meiss M, 1972 The Visconti Hours (New York: George Braziller)

Stoichita V I, 1997 A Short History of the Shadow (London: Reaktion).

\section{Acknowledgments}

Thanks to Patrick Cavanagh, Jayme Jacobson, Pascal Mamassian, Milena Nuti and Alessandro Pignocchi for comments; Jelena Arsenic and Svetislava Isakov for help with the pictures. 